\title{
Orthodontics and COVID-19 pandemic - a review
}

\author{
Aurelia Magdalena Enache ${ }^{1}$, Dana Festila ${ }^{2}$ \\ ${ }^{1}$ Department of Orthodontics and Dentofacial Orthopedics, \\ "Carol Davila" University of Medicine and Pharmacy, Bucharest, Romania \\ ${ }^{2}$ Department of Orthodontics and Dentofacial Orthopedics, \\ "Iuliu Hatieganu" University of Medicine and Pharmacy, Cluj Napoca, Romania
}

\begin{abstract}
Severe acute respiratory syndrome coronavirus 2 (SARS-CoV-2) is a highly infectious disease with a major impact on orthodontic practice regarding the risk of control transmission of COVID 19. Due to the particularities of orthodontic treatment which includes return-visits of the patients for a long period of time, the number of patients per day, dental aerosols-generated procedures risk, management considerations should be constant in order to update practice to the scientific evidence. The aim of this critical narrative review of the literature is to highlights the latest scientific data in order to control the transmission of SARS-CoV-2 infection in the orthodontic practice.
\end{abstract}

Keywords: COVID-19, pandemic, orthodontic practice, aerosols-generated proccedures

\section{INTRODUCTION}

Severe acute respiratory syndrome coronavirus 2 (SARS-CoV-2) has spread rapidly since December 2019, when it emerged in the city of Wuhan, China and it was declared a pandemic by the World Health Organization, on 11 March 2020 (1). Being a highly infectious disease (COVID-19), it has had a major impact on heath systems worldwide. Due to the transmission modalities of SARS-CoV-2 and the particularities of dental treatment procedures, including orthodontics many preventive measures were taken around the world, like quarantine and lockdown, in order to control the spread of COVID-19 and mortality.

The three most common symptoms of COVID-19 are fever, dry cough and dyspnea. Additional symptoms include headaches, weakness, fatigue, nausea, vomiting, diarrhea, anosmia, ageusia but severe patients rapidly progress to respiratory distress syndrome, septic shock, metabolic acidosis, coagulation dysfunction and multiple organ failures (2-5).
Regarding the transmission of the SARS-CoV-2, although not fully understood, the studies confirmed the transmission from humans to humans by two modalities: direct inhalation of airborne virus contained in infectious droplets produced from an infected patient, or indirect transfer to the mouth, nose or eyes from surfaces contaminated with infected salivary or respiratory secretions or the settling of the airborne virus $(4,6,7)$. Due to recent evidence of SARS-CoV-2 transmission through presymptomatic, asymptomatic and symptomatic patients (7-12) children being less symptomatic than adults (7) and the difficulty of diagnose and isolate these patients in time, controlling the transmission of COVID-19 in orthodonthic practice is of utmost importance. In the incubation period of COVID which is about 3-14 days with an average of 5-6 days, but it has also been reported as long as 24 days, the patients could release a large amount of the virus (13-16). Consequently the aim of this narrative review of the literature is to highlights latest evidence-based data in order to 
reduce the risk of SARS-CoV-2 transmission in orthodontic practice.

\section{DENTAL AGPS IN ORTHODONTIC PRACTICE}

It is well-known that infection control in dental practice (including orthodontics) has been our concern for a long period of time, but for COVID-19 pandemic period the previous standard measures might not be enough to protect both orthodontic staff and patient from this disease. The impact of social distancing on scheduling orthodontic treatments increases patient anxiety of prolonged orthodontic treatment (17) and because of the safety of our orthodontic practice is our responsibility we have to establish a strict protocol on a scientific bases. The additional measures of first importance should take into consideration COVID-19 screening before any appointment in orthodontic office, personal protective equipment (PPE) and dental AGPs.

Aerosols generated procedures (AGPs) in dental practice has the most important role in infection transmission and for this reason all efforts have to be done in order to minimize them.

The term of aerosol was introduced by Fredderick G. Donnan, being defined as a suspension system of liquid or solid particle in a gas (18).

Airborne viruses, approximately $0.1 \mu \mathrm{m}$ come from droplets from coughing, sneezing, talking, exhaling breath or mechanical aerosolization of infected salivary or respiratory secretions generated by different medical and dental proceduresaerosols generated procedures (AGPs). The droplets can vary, as dimension, from small droplets (droplets nuclei) which are less then $5 \mu \mathrm{m}$ to large droplets, bigger than $5 \mu \mathrm{m}$. The small droplets and aerosols are more sussceptible to be inhaled deep into the lung.

The pathogenity of dental aerosols depend on the bacterium or virus load in compressed air or water-spray spatter mixed-up with blood, plaque, tooth material, calculus, saliva debris produced during routine dental practice (19). Rautemaa found a mean bacterial load range of 1-3.9 CFUs (colony forming units) after dental AGPs with the highest value at $1.5 \mathrm{~m}$ from oral cavity (20).

Dental AGPs in orthodontic practice are considered of limited extent compared with dental practice, but we should not neglect their impor- tance in infection control $(4,21)$. The procedures like photographing, impression taking, digital oral scanning, that may stimulate gagging reflex and those using handpieces, air-water syringes, ultrasonic scalers, airflow device or sandblaster are dental AGPs for orthodontics, the latest being mostly involved in bonding and debonding of the fixed appliances. Although recent literature recommends aligner therapy for orthodontic treatment as a safer modality for this pandemic period (22) it should be mentioned that removal of the attachments at the end of treatment or attachment replacement during treatment are dental AGPs, that should be taken into consideration.

The pathogenic state of aerosols produced during bracket debonding procedure followed by enamel clean-up with high-speed handpiece and water cooling is higher than standard orthodontic procedures with a mean difference of 49.2 CFUs (21). Bacterial load of aerosols produced by dental AGPs is significantly raised in $5 \mathrm{~min}$ after the procedure (19). Oral streptococci in aerosols generated by dental AGPs dissipate within 30 min of their peak concentration, but it must be mentioned that streptococci are 10 times larger than SARS-CoV-2, which may increase the presence of the virus in the aerosols (23).

\section{MODALITIES TO REDUCE THE EFFECT OF DENTAL AGPS IN COVID-19 TRANSMISSION DURING ORTHODONTIC TREATMENT}

A well-known measure to reduce the pathogenic state of the bioaerosols produced in dental AGPs is preprocedural mouthrinse. A recent meta-analysis study (24) shown that there is a moderate evidence that preprocedural mouthrinses significantly reduce the number of microorganisms in the dental aerosols. Preprocedural mouthrinses for $30 \mathrm{~s}$ to $1 \mathrm{~min}$ with chlorhexidine (CHX) $0.2 \%$ tempered at $47^{\circ}$ has been documented to have anti-microbiologic effect against bacteria on the human dental plaque (43). National Health Comission People Republic of China recommended preprocedural mouthrinses with hydrogen peroxide $1 \%$ or povidone iodine (PI) $0,2-1 \%$ because of the oxidative action on lipid shell membrane of the virus. A recent critical review has concluded that there is not sufficient scientific evidence to support the recommendations to manage the risk of infection in the dental 
office by preprocedural mouthrinses, although some ingredients in the antiseptic mouthrinses have antiviral properties (7). In accordance with CDC (Centers for Disease Control and Prevention) guidelines preprocedural mouthrinses with antimicrobial agent (CHX, PI, essential oils) may reduce the oral microorganisms load in bioaerosols and spatter generated during dental procedures (7).

Another preventive measure is to wash and spray the removable appliances, including aligners with $75 \%$ alcohol or chlorine-containing disifectants before adjustment, because they are in direct contact with saliva and mucosa and can be potential media for transmission of SARS-CoV-2 (5). The inserting or removal of the removable appliances could be also dental AGPs due to gagging reflex.

As it was mentioned before two important orthodontic AGPs are bonding and debonding. Regarding bonding procedures, enamel etching, rinsing after enamel etching and type of bonding material are factors that should be taken into consideration in order to reduce AGPs. Because thick consistencies etchant acid gels for enamel conditioning needs high water and/or spray pressure to rinse off, low viscosity gels or even liquid etchant acid are recommended in order to reduce spatter and droplets formations (19). Using self-etching primer instead of etchant gel and bonding resin is another option that can minimize AGPs. In order to avoid enamel conditioning and subsequent dental AGPs, in this pandemic period, for brackets bonding, glass-ionomer cements are recommended. A simplified bonding technique, a less susceptibility to moisture in the oral cavity and faster debonding with reduced risk of enamel damage are the advantages of glass-ionomer cemets for brackets bonding procedure. Indirect bonding can also reduce the amount of bioaerosols generated because it is not so time consuming as direct bonding.

Brackets debonding at the end of orthodontic treatment or attachments removal in aligner therapy as dental AGP has two major components-griding of the remnant bonding material from the teeth and subsequent cleaning of the teeth, which increase hazardous concern for orthodontic team and patients especially in this COVID-19 pandemic period. Although the amount of bioaerosols produced during debonding is not exactly known and is unknown for virus and virus particles $(7,25,26)$, composite grinding produces the most particulate aerosols of any other dental AGP tested in situ, airborne particulates increasing approximately 6-folds compared with backgraound levels (27). Regarding of the bonding material grinding duration, amount of aerosolized dust, droplets and bioaerosols should be minimized and all of these are directly correlated with the type of the rotary instruments, slow versus high speed handpieces and moisture conditions. Eliades recommends the use of carbide tungsten bur under water cooling conditions in order to enhance the cutting efficiency, reduce procedure duratiom and amount of aerosolized nanoparticles (19). If an individualized bracket is debonded during orthodontic treatment hand instruments for bonding material removal is indicated as an effective strategy for this pandemic period. Studies that assessed the production of aerosols containing bonding adhesive and enamel particulates during orthodontic adhesive removal from humans teeth under laboratory conditions $(7,28,29,30)$ concluded that slower speed and water spray reduce the amount of particulate aerosols produced. In case of composite grinding under water usage conditions the bioaerosols generated nanoparticles are trapped into droplets that can not penetrate the epithelial bronchial barriers and get further into the lung(19).

In order to minimize the role of dental AGPs in infection control, regarding bonding and debonding efforts are made to find bonding materials that doesn't require prior enamel conditioning, like so-called bio-mimetic materials (gekkonidae lizards) and to reduce the amount of composite remnants after bracket removal with direct correlation to dust and particulate generation, bioaerosols and droplets formation and their pathogenity (as BPA-free adhesives) (19).

Air and environment disinfection is another modality. to reduce bioaerosols load in orthodontic practice and to prevent the spread of SARS-CoV-2 infection. Plasma air sterilizers or sterilizers using UVC light can be used during activity in the presence of humans, especially in the working and waiting area, but natural ventilation is a simple and effective way of air purification. Microbial colonies reduced by $77.3-79.3 \%$ in the first $30 \mathrm{~min}$ and up to $96.4-99.3 \%$ in the first $75 \mathrm{~min}$ (31). The disadvantages of natural ventilation is the increased period of time between patients, that will reduce 
the number of patient a day and consequently will increase the number of working days and eventually the treatment time (4). Regarding how much time should be allocated between patients when dental aerosol-generated procedure is performed there is not enough scientific evidence, because the risk of dental AGPs for presymptomatic and asymotomatic patients cannot be quantifiable. An important factor that has to be taken into consideration is the rates of air exchange of air conditioning system. It can be used also high-volume extraoral evacuation (HVE), as an adjunctive measure to reduce the risk of SARS-CoV-2 transmission during orthodontic debonding, but it's efficiency remains unclear (7). Although there are new evacuation devices their efficacy in reducing mechanically-generated aerosols required ongoing research (32). CDC recommends portable high-efficiency particulate air (HEPA) filtration system properly positioned (which is still under ongoing research) in order to lessen the risk of COVID-19 transmission via bioaerosols during dental AGPs, because there is ample evidence that HEPA filtration system reduces the concentration of the airborne particles with a size range similar to SARS-CoV-2, but not direct evidence for reducing the viable virus in the operatory during dental AGPs.

\section{PPE AND SOCIAL DISTANCING IN ORTHODONTIC PRACTICE DURING COVID-19 PANDEMIC}

As previously discussed the risk of transmission of SARS-CoV-2 in orthodontic practice is not just during dental AGPs, but also through bioaerosols generated from coughing, sneezing, exhaling during standard procedures and PPE (face mask, gloves, face shields, gowns) is part of the system that protects doctors, staff and patients. It has to be noted that PPE is very uncomfortable in day-today practice and affects psychological interaction between doctors and patients, which isn't negligible in the orthodontic practice. An important component of PPE is face mask and the recommendation for dental AGPs is high-filtration mask (N95/
FFP2), although there are reports of headache of the health care personal during prolonged use of N95 respirators $(33,34)$. A systematic review found no difference between N95 respirator and surgical masks in health care workers exposed to acute viral respiratory infections, with a low or very low evidence due to the heterogeneity research method (35), but there is no research about N95 respirator protection against COVID-19 transmission.

The COVID-19 pandemic, due to the need of social distancing, changed the scheduling pattern, reducing the number of patients a day although, as previously mentioned, there are no scientific data for how much time should be between patients in order to reduce the risk of infection transmission and it is advisable to use telemedicine for those orthodontic procedures that can take advantage of it (screening, consultation, some aligner therapy visits etc). Consequently, the working days and the number of working hours per day for the same number of patients increased in orthodontic practice.

A new approach of orthodontic treatment should be considered in this pandemic period, from the psychological and ergonomic prospective. It is important to assess the best time to start orthodontic treatment or to postpone it. From psychological point of view orthodontic treatment is important for patients but actual economic conditions have to consider for some specific treatment. Some recent studies recommend aligner therapy for the pandemic period, due to advantages of less visits and emergencies and the reduced mental distress caused to the patients in the early stage of the pandemic, during quarantine, but the costs of oral scanner and treatment may be a limitation of this technique.

\section{CONCLUSION}

The COVID-19 pandemic has a long-lasting impact on the orthodontic practice and the best practice should be always correlated with the scientific evidence from the ongoing research.

Conflict of interest: none declared Financial support: none declared 


\section{REFERENCES}

1. World Health Organization -Archived-WHO Timeline-COVID 19, available at: https://www.who.int/news/item/27-04-2020-whotimeline-covid-19.

2. Guan WJ, Zhong NS. Clinical Characteristics of Covid-19 in China. Reply. N Engl J Med. 2020;382(19):1861-1862.

3. Lovato A, de Filippis C. Clinical Presentation of COVID-19: A Systematic Review Focusing on Upper Airway Symptoms. Ear Nose Throat J. 2020;99(9):569-576.

4. Martina S, Amato A, Rongo R, Caggiano M, Amato M. The Perception of COVID-19 among Italian Dentists: An Orthodontic Point of View. Int J Environ Res Public Health. 2020;17(12):4384. Published 2020 Jun 18.

5. Guo Y, Jing Y, Wang Y, et al. Control of SARS-CoV-2 transmission in orthodontic practice. Am J Orthod Dentofacial Orthop. 2020; 158(3):321-329.

6. Scheuch G. Breathing Is Enough: For the Spread of Influenza Virus and SARS-CoV-2 by Breathing Only. J Aerosol Med Pulm Drug Deliv. 2020;33(4):230-234.

7. Marshall S, Duryea M, Huang G, et al. COVID-19: What do we know?. Am J Orthod Dentofacial Orthop. 2020;158(5):e53-e62.

8. Wölfel R, Corman VM, Guggemos W, et al. Virological assessment of hospitalized patients with COVID-2019 [published correction appears in Nature. 2020 Dec;588(7839):E35]. Nature. 2020; 581(7809):465-469.

9. Yu P, Zhu J, Zhang Z, Han Y. A Familial Cluster of Infection Associated With the 2019 Novel Coronavirus Indicating Possible Person-to-Person Transmission During the Incubation Period. $J$ Infect Dis. 2020;221(11):1757-1761.

10. Kimball A, Hatfield KM, Arons M, et al. Asymptomatic and Presymptomatic SARS-CoV-2 Infections in Residents of a Long-Term Care Skilled Nursing Facility - King County, Washington, March 2020. MMWR Morb Mortal Wkly Rep. 2020;69(13):377-381. Published 2020 Apr 3.

11. Byambasuren $\mathrm{O}$, Cardona $\mathrm{M}$, Bell $\mathrm{K}$ et al. Estimating the extent of true asymptomatic COVID-19 and its potential for community transmission: systematic review and meta-analysis. medRxiv. 2020

12. Pan $X$, Chen $D$, Xia $Y$, et al. Asymptomatic cases in a family cluster with SARS-CoV-2 infection. Lancet Infect Dis. 2020;20(4):410-411.

13. Backer JA, Klinkenberg D, Wallinga J. Incubation period of 2019 novel coronavirus (2019-nCoV) infections among travellers from Wuhan, China, 20-28 January 2020. Euro Surveill. 2020; 25(5):2000062.

14. Li Q, Guan X, Wu P, et al. Early Transmission Dynamics in Wuhan, China, of Novel Coronavirus-Infected Pneumonia. N Engl J Med. 2020;382(13):1199-1207.

15. Guan WJ, Ni ZY, Hu Y, et al. Clinical Characteristics of Coronavirus Disease 2019 in China. N Engl J Med. 2020;382(18):1708-1720.

16. Ganyani T, Kremer $C$, Chen $D$, et al. Estimating the generation interval for coronavirus disease (COVID-19) based on symptom onset data, March 2020. Euro Surveill. 2020;25(17):2000257.

17. Pires AC, Souza SLX de, Cavalcanti AL. Panorama of Orthodontics after COVID-19: A critical literature review. RSD [Internet]. 2020 [cited 2021 Jan.7];9(9):e924998103. Available at: https://rsdjournal. org/index.php/rsd/article/view/8103.

18. Hinds WC. Aerosol Technology: Properties, Behavior, and Measurement of Airborne Particles. 2nd ed. Hoboken: WileyInterscience, 1999.
19. Eliades T, Koletsi D. Minimizing the aerosol-generating procedures in orthodontics in the era of a pandemic: Current evidence on the reduction of hazardous effects for the treatment team and patients. Am J Orthod Dentofacial Orthop. 2020;158(3):330-342.

20. Rautemaa R, Nordberg A, Wuolijoki-Saaristo K, Meurman JH. Bacterial aerosols in dental practice - a potential hospital infection problem?. J Hosp Infect. 2006;64(1):76-81.

21. Toroğlu MS, Haytaç MC, Köksal F. Evaluation of aerosol contamination during debonding procedures. Angle Orthod. 2001; 71(4):299-306.

22. Kaur H, Kochhar AS, Gupta H, Singh G, Kubavat A. Appropriate orthodontic appliances during the COVID-19 pandemic: A scoping review. J Oral Biol Craniofac Res. 2020;10(4):782-787.

23. Bennett AM, Fulford MR, Walker JT, Bradshaw DJ, Martin MV, Marsh PD. Microbial aerosols in general dental practice. Br Dent $J$. 2000;189(12):664-667.

24. Marui VC, Souto MLS, Rovai ES, Romito GA, Chambrone L, Pannuti CM. Efficacy of preprocedural mouthrinses in the reduction of microorganisms in aerosol: A systematic review. J Am Dent Assoc. 2019;150(12):1015-1026.e1.

25. Zemouri $C$, de Soet $H$, Crielaard W, Laheij A. A scoping review on bio-aerosols in healthcare and the dental environment. PLoS One. 2017;12(5):e0178007.

26. Iliadi A, Koletsi D, Eliades T, Eliades G. Particulate Production and Composite Dust during Routine Dental Procedures. A Systematic Review with Meta-Analyses. Materials (Basel). 2020;13(11):2513.

27. Polednik B. Aerosol and bioaerosol particles in a dental office. Environ Res. 2014;134:405-409.

28. Ireland AJ, Moreno T, Price R. Airborne particles produced during enamel cleanup after removal of orthodontic appliances. $A m \mathrm{~J}$ Orthod Dentofacial Orthop. 2003;124(6):683-686.

29. Day CJ, Price R, Sandy JR, Ireland AJ. Inhalation of aerosols produced during the removal of fixed orthodontic appliances: a comparison of 4 enamel cleanup methods. Am J Orthod Dentofacial Orthop. 2008;133(1):11-17.

30. Vig P, Atack NE, Sandy JR, Sherriff M, Ireland AJ. Particulate production during debonding of fixed appliances: Laboratory investigation and randomized clinical trial to assess the effect of using flash-free ceramic brackets. Am J Orthod Dentofacial Orthop. 2019;155(6):767-778.

31. Fu Shaw $L$, Chen $\mathrm{IH}$, Chen CS, et al. Factors influencing microbial colonies in the air of operating rooms. BMC Infect Dis. 2018;18(1):4.

32. Matava C, Collard V, Siegel J, et al. Use of a high-flow extractor to reduce aerosol exposure in tracheal intubation. $\mathrm{Br} \mathrm{J}$ Anaesth. 2020; 125(4):e363-e366.

33. Lim EC, Seet RC, Lee KH, Wilder-Smith EP, Chuah BY, Ong BK. Headaches and the N95 face-mask amongst healthcare providers. Acta Neurol Scand. 2006;113(3):199-202.

34. Ong JJY, Bharatendu C, Goh Y, et al. Headaches Associated With Personal Protective Equipment - A Cross-Sectional Study Among Frontline Healthcare Workers During COVID-19. Headache. 2020; 60(5):864-877.

35. Bartoszko JJ, Faroogi MAM, Alhazzani W, Loeb M. Medical masks vs N95 respirators for preventing COVID-19 in healthcare workers: A systematic review and meta-analysis of randomized trials. Influenza Other Respir Viruses. 2020;14(4):365-373. 